prophylaxis (PEP) can be variable and costly, PrEP may be a more effective consideration.

Methods We conducted an anonymous online questionnaire assessing knowledge, attitudes and beliefs about PEP, PrEP and HIV risk among medical students at a UK medical school (GKT School of Medical Education) over 6 weeks. Data was collected using the SurveyMonkey ${ }^{\mathrm{TM}}$ platform.

Results The response rate was 351/2295 (15.3\%). 312/351 (89\%) would consider PrEP as an alternative to PEP for their elective if prescribed by a healthcare professional. Of these, 183/312 (59\%) would be comfortable obtaining PrEP online. Concerns were around the quality of the medication 84/129 (65\%), side effects 57/129 (44\%), low perceived risk of HIV exposure 43/129 (33\%), efficacy of PrEP 38/129 (29\%), drug resistance 26/129 (20\%), adherence 19/129 (15\%) and cost 15/129 (12\%). 276/351 (79\%) were aware of PrEP. However, students reported limited knowledge with an average knowledge score of 2.65/5. 48/351 (14\%) planned on taking a supply of PEP on elective. 88/351 (25\%) were visiting areas with a high HIV prevalence of whom 59 intended to engage in a high-risk specialty. Of the highest risk students, 40/59 (68\%) were aware of the high HIV prevalence but only 14/59 (24\%) were planning on taking PEP.

Conclusion Medical Students are open to the idea of considering PrEP to reduce their risk of HIV through occupational or sexual exposure. Students report low knowledge of PrEP, and variable knowledge of their individual risks and mitigations. This research indicates a need for robust educational interventions highlighting the benefits of PrEP among medical students and their healthcare advisers.

Disclosure No significant relationships.

\section{P424 HIGH PREVALENCE AND INCIDENCE OF BACTERIAL STIS IN YOUNG WOMEN AT HIGH RISK OF HIV PRIOR TO PREP SCALE-UP IN KENYA}

${ }^{1}$ Jenell Stewart, ${ }^{2}$ Victor Omollo, ${ }^{2}$ Josephine Odoyo, ${ }^{3}$ Lara Kidoguchi, ${ }^{3}$ Jennifer Morton, ${ }^{3}$ Rachel Johnson, ${ }^{1}$ Connie Celum, ${ }^{4}$ Jared Baeten, ${ }^{5}$ Elizabeth Bukusi. 'University of Washington, Medicine, Seattle, USA; ${ }^{2}$ KEMRI, Nairobi, Kenya; ${ }^{3}$ University of Washington, Seattle, USA; ${ }^{4}$ University of Washington, Medicine, Global Health, Epidemiology, Seattle, USA; ${ }^{5}$ Kenya Medical Research Institute, Nairobi, Kenya

10.1136/sextrans-2019-sti.510

Background Young women account for more than half of new HIV infections in sub-Saharan Africa and are a target population for HIV pre-exposure prophylaxis (PrEP). Although it protects against HIV acquisition, PrEP lacks protection against bacterial sexually transmitted infections (STIs). High rates of STIs have been reported among PrEP-using men who have sex with men (MSM) in high income countries. STI rates among African adolescent girls and young women (AGYW) have not been well-described.

Methods POWER is a multi-site PrEP implementation science project among AGYW aged 16-25 in Kisumu, Kenya (beginning August 2017) as well as South Africa; we describe results from the Kenyan cohort here. At enrollment, participants are offered PrEP; follow-up visits are at month-one and then quarterly for 36 months with STI testing at enrollment before PrEP initiation and every 6 months. Urine samples were tested for Chlamydia trachomatis and Neisseria gonorrhoeae by nucleic acid amplification (NAAT) and participants with infections returned to the research site for treatment.
Results To date, 708 Kenyan women have enrolled in POWER with $17 \%$ prevalence of C. trachomatis and $8 \%$ of N. gonorrhoeae at enrollment. Among the 65 women with 6-month follow-up in Kenya; the incidence of C. trachomatis and $N$. gonorrhoeae were 40.0 and 12.3 per 100 person-years.

Conclusion In a PrEP scale-up cohort among Kenyan AGYW, STI prevalence and incidence were very high, which predict substantial morbidity. These STI rates are comparable to those seen among PrEP-using MSM in the US after years of rising rates; Kenya has had only early PrEP roll-out and thus these findings are largely prior to community delivery of PrEP. It is not yet known if STI rates will increase among with PrEP use in Kenya, and PrEP roll-out programs provide a crucial opportunity to address both HIV and STI epidemics simultaneously. Disclosure No significant relationships.

\section{P425 CAPTURING MISSED OPPORTUNITIES FOR PREP PRESCRIPTION IN PATIENT DIAGNOSED WITH OTHER STIS}

${ }^{1}$ Stephanie Mclaughlin*, ${ }^{1}$ Robert Pitts, ${ }^{2}$ Farzana Kapadia, ${ }^{1}$ Richard Greene. ${ }^{1}$ New York University School of Medicine, Internal Medicine, New York, USA; ${ }^{2}$ New York University, Epidemiology, New York, USA

10.1136/sextrans-2019-sti.511

Background Sexually transmitted infection (STI) testing in hospital-based settings represents an opportunity to intervene and prescribe pre-exposure prophylaxis (PrEP) to prevent HIV infections.

Methods Electronic health records of patient visits at NYC Health + Hospitals $(\mathrm{H}+\mathrm{H}) /$ Bellevue between 1/1/14-7/30/17 were queried for positive STIs (gonorrhea (GC) and chlamydia (CT) $\&$ syphilis) results by hospital location and time. Visit data also included: patient demographic characteristics and PrEP prescriptions. Generalized estimating equations using a logit link, to account for repeated within patient measures, were used to explore the relationship between having a medical follow-up visit in $\leq 30$ and $\leq 90$ days following STI testing, which represents an opportunity to provide PrEP prescription, and demographic factors, controlling for key confounders.

Results A total of 1,169 HIV-negative patients with $1+$ STI diagnosis contributed 1,275 visits, of whom 700 (58\%) were female with mean age of $32 \mathrm{yrs}(\mathrm{SD}=12.3)]$ and $532(42 \%)$ were male with mean age of 44 yrs (SD 16.3). The majority of patients were Black (40\%) or Hispanic/Latino (50\%). In this sample, only 27 patients received PrEP. Overall, chlamydia was the most common (135/11\%), followed by syphilis (476/ $38 \%)$ and gonorrhea (135/11\%). Two-thirds of patients with a + STI diagnosis originated from the ED (33\%) and OB/GYN clinics (32\%); an additional 11\% were diagnosed in Medicine clinics. $78 \%$ of patients did not have follow up $\leq 30$ days after + STI diagnosis. In adjusted analyses, the adjusted OR for follow up $\leq 30$ days after + STI diagnosis was lower for Black patients [0.39 $(95 \% \mathrm{CI} 0.21-0.72, \mathrm{p}<0.01)]$ and higher for patients $\geq 45$ years old 2.20 [(95\%CI 1.16-4.19, $\mathrm{p}=0.02)$ ].

Conclusion STI testing at a major, publicly-funded hospital within NYC H\&H is an opportunity to discuss and prescribe PrEP. However, our findings suggest that there are significant missed opportunities for linkage to care after a +STI diagnosis and PrEP initiation, especially in the ED among young Black patients.

Disclosure No significant relationships. 